\title{
Knowledge and Risks of Zoonotic Diseases among Livestock Farmers in Budgam District of Kashmir Valley, India
}

\author{
Insha Mir* and Abdul Hai Bhat \\ Department of Veterinary \& Animal Husbandry Extension Education \\ Sher-e-Kashmir University of Agricultural Sciences andTechnology of Kashmir, India \\ *Corresponding author
}

\begin{abstract}
A B S T R A C T
Majority of the respondents belonged to middle age group 30-50 years $(65.94 \%)$, mostly were illiterate $(81.88 \%)$, farming was the main occupation $(61.88 \%)$, and had their herd size up to 4 animals $(96.56 \%)$. Contact with inter personal communication sources was found medium among (59.19\%) respondents , participation in extension activities was low found in (99.69\%), and mass media support was also found low among (92.19\%). Regarding transmission of zoonotic diseases $(48.13 \%)$ were aware. About $(15.94 \%)$ of livestock farmers were aware of rabies, brucellosis, tuberculosis, anthrax, bird flu, echnicoccosis and swine flu. About $(15.31 \%)$ agree of the transmission of zoonotic diseases to human beings through contaminated milk, meat, egg, air, feed, or through contact with infected animals. About (37.50\%) of respondents had knowledge regarding risks of zoonotic diseases from various activities related with livestock rearing. Overall knowledge level was found low to medium level categories, whereas overall risk level was medium among livestock farmers.
\end{abstract}

\section{Keywords}

Knowledge, Risk, Zoonotic disease

Article Info

Accepted:

22 March 2020

Available Online:

10 April 2020

\section{Introduction}

Zoonoses are defined as those diseases that are naturally transmitted between people and vertebrate animals. Zoonoses constitute a diverse group of viral, bacterial, ricketssial, fungal, parasitic, and prion disease with a variety of animal reservoirs, including wild life, livestock, pet animals, and birds (Nkuchia et al., 2007). The transmission may occur through direct contact with the animal, through vectors such as mosquitoes, fleas or ticks or through food or water contamination. Zoonotic diseases have both direct and indirect effects on livestock health and its production (Smiths and Cutler, 2004).

Zoonotic diseases cause morbidity and mortality in people, and is also imposing significantly losses to the livestock sector that account about $75 \%$ all emerging pathogens. When it comes to the reality people who are 
from farming community are often exposed to zoonotic risks. The agriculture and animal husbandry are the two main occupations which expose farmers to a number of dreaded zoonotic diseases and their potentially disastrous impact on human health is a growing concern around the globe (Woolhouse and Sequeria, 2005) annually.

Since zoonotic infections remain age-long, many factors including increase contact between animals and humans continue to play a key role in their emergence and persistence. Environmental changes due to natural calamities and manmade activities, customs and traditions followed by different people in various countries, increase in human population, urbanization and increased movement of wild animals towards human habitations due to deforestation, are some of the factors responsible for spread of the zoonotic diseases.

In most of the under developed and developing countries farming practices, low education level, culture and eating habits, presence of reservoir population, inadequate disease control programmes and lack of knowledge about disease burden have been mostly reported to be associated with persistence of zoonotic diseases (Asbjer, 2009).Lack of knowledge and awareness with regard to zoonotic diseases is one of the most important reasons for the frequent outbreaks of zoonotic diseases in people in general.

\section{Materials and Methods}

Budgam district was purposefully selected for the study. A list of 8 blocks chosen for the study was randomly selected. From the selected 8 blocks, randomly 2 villages were selected from each block. Thus, a total 16 villages were randomly selected in all. From each randomly selected village, 20 respondents were taken into confidence and total respondents were 320 . The data was tabulated and analyzed using statistical package for the Social Science (SPSS) version 20 developed by IBM Company, USA.

\section{Results and Discussion}

\section{Socio-personal and socio-economic characteristics of livestock farmers}

Regarding socio- personal characteristics, it was found that majority of the respondents were from middle age group though probably this age group has the enthusiasm of youth and experience of old which can be said to be a good blend for having well knowledge and awareness.

By analysis on gender it was found more males were involved in livestock rearing practices. Similar findings were observed by Folayan (2013) and Oluwafeni (2015) who reported that livestock rearing is a gender sensitive (a male domain) and the majority were active in their middle ages.

The community status depends upon the educational level of the members and this study revealed that most of the respondents $(81.88 \%)$ were illiterate which clearly indicates that livestock owners had less knowledge and awareness about zoonotic diseases and associated hazards. The findings were also in agreement with the findings of Mahdi and Ali (2002) studied that majority of illiterate and middle aged group was fatal for zoonotic diseases.

It was also revealed from the study that majority of the farmers were having marginal land and there is a high significant variation regarding land holdings. Probably this is the reason why along with agriculture the farmers keep livestock as supportive income. 
Further sustained when we have a look into the data pertaining to livestock possession which revealed that $(96.56 \%)$ had a range of 01-04 livestock possession and statistically there is a high significant variation. In agreement with Ward and Moon (1996) found that livestock possession showed positive and significant relation with entrepreneurial behavior.

\section{Communication patterns with regard to dissemination of knowledge related to zoonotic diseases among livestock farmers}

The analysis of success and failure of rural development programmes often centers on the identification of communication breakthrough or communication break-down. Handled well the communication process will enable to change knowledge, awareness, behavior and attitude of millions of rural people. To ascertain the existing communication patterns prevalent in Budgam district particularly regarding knowledge, awareness and risks regarding zoonotic diseases via. Interpersonal communication sources, participation in extension activities, and mass media support.

So the contact with interpersonal sources by livestock farmers were depicted in this study and the livestock farmers by and large had either frequent, occasional or rare contacts with different inter personal communication sources and it was found that $(39.92 \%)$ were having frequent contact $(59.19 \%)$ were occasionally connected followed by $(0.89 \%)$ rarely and found statistically that there is nonsignificant difference. And when compared with Yadav et al., (2006) assessed that information gathering as well as problem solving process was mostly linked in this fashion and the impact of interpersonal communication sources in transfer of knowledge and awareness regarding zoonotic importance.
Further enquires were held to ascertain their participation in various extension activities. Six activities were selected for the purpose viz. training, meeting, demonstration, field day, Kisan mela, visit to university /Research station/ KVK. And from the study it was revealed that $99.69 \%$ rarely participated in any of the above activities. Also with the agreement with Niyaki and Allahyari (2010) and Rahman and Gupta (2014) found that participation in extension activities will improve in knowledge gain.

This shows clearly the failure on the part of Animal Husbandry Department, University and other agencies for not organizing enough training programmes for the awareness of livestock farmer. Since training improves knowledge, awareness and skill, it is very essential that regular training programmes should be organized to spread awareness regarding zoonotic diseases among livestock farmers. Mass media which avail as the best means of information includes radio which is being available and popular amongst livestock farmers can be a good medium for transfer of awareness, T.V which is the latest and effective tool for transfer of knowledge, also use of extension literature which is found beneficial to literate livestock farmers, film shows/video shows and non-projected visual aids which avail to disseminate knowledge in a clear way.

And from the present study it was found that only $(7.81 \%)$ of the respondents occasionally use mass media as means of communication with regard to zoonotic diseases and statistically found non-significant variation. When compared with Ahn et al., (1981) found that radio and T.V. programmes can change the attitude level of farmers in Korea regarding zoonotic diseases. Also from Huang et al., (2014) revealed that T.V and newspapers helped regarding awareness of zoonotic disease occurrence (Table 1-12). 
Table.1 Distribution of respondents according to age

\begin{tabular}{|c|c|c|c|c|c|c|c|c|c|}
\hline \multirow{2}{*}{$\begin{array}{c}\text { Age } \\
\text { (Years) }\end{array}$} & \multicolumn{8}{|c|}{ Blocks } & \multirow[t]{2}{*}{ Pooled } \\
\hline & Budgam & Beerwah & Chadoora & Khansahib & Khag & B K Pora & Narbal & Nagam & \\
\hline $\mathbf{N}$ & 40 & 40 & 40 & 40 & 40 & 40 & 40 & 40 & 320 \\
\hline $\begin{array}{c}<30 \\
\text { years }\end{array}$ & 2 & 7 & 10 & 6 & 8 & 4 & 5 & 6 & 48 \\
\hline (Young) & $(5.00)$ & (17.50) & $(25.00)$ & $(15.00)$ & (20.00) & $(10.00)$ & $(12.50)$ & $(15.00)$ & $(15.00)$ \\
\hline $\begin{array}{l}30-50 \\
\text { years }\end{array}$ & 27 & 28 & 23 & 29 & 22 & 28 & 27 & 27 & 211 \\
\hline (Middle) & $(67.50)$ & $(70.00)$ & $(57.50)$ & $(72.50)$ & $(55.00)$ & $(70.00)$ & $(67.50)$ & $(67.50)$ & $(65.94)$ \\
\hline$>50$ years & 11 & 5 & 7 & 5 & 10 & 8 & 8 & 7 & 61 \\
\hline (Old) & $(27.50)$ & $(12.50)$ & $(17.50)$ & $(12.50)$ & $(25.00)$ & $(20.00)$ & $(20.00)$ & $(17.50)$ & (19.06) \\
\hline Mean \pm SD & $42.1 \pm 8.77$ & $37.3 \pm 8.71$ & $38.52 \pm 9.48$ & $39.1 \pm 8.56$ & $39.97 \pm 10.30$ & $39.55 \pm 10.14$ & $40.15 \pm 9.10$ & $38.8 \pm 9.29$ & $39.43 \pm 9.30$ \\
\hline
\end{tabular}

(Figures in parenthesis indicate percentage, $\mathrm{n}$ indicate sample size $)(\mathrm{P}$ value $=0.539)$

Table.2 Distribution of respondents according to their gender

\begin{tabular}{|c|c|c|c|c|c|c|c|c|c|}
\hline Gender & \multicolumn{9}{|c|}{ Blocks } \\
\hline & Budgam & Beerwah & Chadoora & Khansahib & Khag & B K Pora & Narbal & Nagam \\
\hline N & $\mathbf{4 0}$ & $\mathbf{4 0}$ & $\mathbf{4 0}$ & $\mathbf{4 0}$ & $\mathbf{4 0}$ & $\mathbf{4 0}$ & $\mathbf{4 0}$ & $\mathbf{4 0}$ & $\mathbf{3 2 0}$ \\
\hline Male & 21 & 25 & 22 & 19 & 22 & 21 & 23 & 25 & 178 \\
\hline & $(52.50)$ & $(62.50)$ & $(55.00)$ & $(47.50)$ & $(55.00)$ & $(52.50)$ & $(57.50)$ & $(62.50)$ & $(55.63)$ \\
\hline Female & 19 & 15 & 18 & 21 & 18 & 19 & 17 & 15 & 142 \\
& $(47.50)$ & $(37.50)$ & $(45.00)$ & $(52.50)$ & $(45.00)$ & $(47.50)$ & $(42.50)$ & $(37.50)$ & $(44.38)$ \\
\hline
\end{tabular}

(Figures in parenthesis indicate percentage, $\mathrm{n}$ indicate sample size $)(\mathrm{P}$ value $=0.047)$ 
Table.3 Distribution of respondents according to their education

\begin{tabular}{|c|c|c|c|c|c|c|c|c|c|}
\hline \multirow[t]{2}{*}{ Education } & \multicolumn{8}{|c|}{ Blocks } & \multirow[t]{2}{*}{ Pooled } \\
\hline & Budgam & Beerwah & Chadoora & Khansahib & Khag & B K Pora & Narbal & Nagam & \\
\hline $\mathbf{N}$ & 40 & 40 & 40 & 40 & 40 & 40 & 40 & 40 & 320 \\
\hline Illiterate & $\begin{array}{c}36 \\
(90.00)\end{array}$ & $\begin{array}{c}33 \\
(82.50)\end{array}$ & $\begin{array}{c}29 \\
(72.50)\end{array}$ & $\begin{array}{c}34 \\
(85.00)\end{array}$ & $\begin{array}{c}35 \\
(87.50)\end{array}$ & $\begin{array}{c}31 \\
(77.50)\end{array}$ & $\begin{array}{c}30 \\
(75.00)\end{array}$ & $\begin{array}{c}34 \\
(85.00)\end{array}$ & $\begin{array}{c}262 \\
(81.88)\end{array}$ \\
\hline Primary & $\begin{array}{c}1 \\
(2.50)\end{array}$ & $\begin{array}{c}4 \\
(10.00)\end{array}$ & $\begin{array}{c}3 \\
(7.50)\end{array}$ & $\begin{array}{c}2 \\
(5.00)\end{array}$ & $\begin{array}{c}2 \\
(5.00)\end{array}$ & $\begin{array}{c}1 \\
(2.50)\end{array}$ & $\begin{array}{c}5 \\
(12.50)\end{array}$ & $\begin{array}{c}3 \\
(7.50)\end{array}$ & $\begin{array}{c}16 \\
(5.00)\end{array}$ \\
\hline Middle & $\begin{array}{c}1 \\
(2.50)\end{array}$ & $\begin{array}{c}0 \\
(0.00)\end{array}$ & $\begin{array}{c}2 \\
(5.00)\end{array}$ & $\begin{array}{c}2 \\
(5.00)\end{array}$ & $\begin{array}{c}1 \\
(2.50)\end{array}$ & $\begin{array}{c}5 \\
(12.50)\end{array}$ & $\begin{array}{c}4 \\
(10.00)\end{array}$ & $\begin{array}{c}3 \\
(7.50)\end{array}$ & $\begin{array}{c}18 \\
(5.63)\end{array}$ \\
\hline High\& 10+2 & $\begin{array}{c}1 \\
(2.50)\end{array}$ & $\begin{array}{c}3 \\
(7.50)\end{array}$ & $\begin{array}{c}4 \\
(10.00)\end{array}$ & $\begin{array}{c}2 \\
(5.00)\end{array}$ & $\begin{array}{c}2 \\
(5.00)\end{array}$ & $\begin{array}{c}3 \\
(7.50)\end{array}$ & $\begin{array}{c}1 \\
(2.50)\end{array}$ & $\begin{array}{c}0 \\
(0.00)\end{array}$ & $\begin{array}{c}16 \\
(5.00)\end{array}$ \\
\hline Graduate \& above & $\begin{array}{c}1 \\
(2.50)\end{array}$ & $\begin{array}{c}0 \\
(0.00)\end{array}$ & $\begin{array}{c}2 \\
(5.00)\end{array}$ & $\begin{array}{c}0 \\
(0.00)\end{array}$ & $\begin{array}{c}0 \\
(0.00)\end{array}$ & $\begin{array}{c}0 \\
(0.00)\end{array}$ & $\begin{array}{c}0 \\
(0.00)\end{array}$ & $\begin{array}{c}0 \\
(0.00)\end{array}$ & $\begin{array}{c}3 \\
(0.94)\end{array}$ \\
\hline
\end{tabular}

(Figures in parenthesis indicate percentage, $\mathrm{n}$ indicate sample size)

Table.4 Distribution of respondents according to their land holdings

\begin{tabular}{|c|c|c|c|c|c|c|c|c|c|}
\hline Land holdings & \multicolumn{8}{|c|}{ Blocks } & Pooled \\
\hline & Budgam & Beerwah & Chadoora & Khansahib & Khag & B K Pora & Narbal & Nagam & \\
\hline $\mathbf{n}$ & 40 & 40 & 40 & 40 & 40 & 40 & 40 & 40 & 320 \\
\hline $\begin{array}{l}\text { Landless } \\
\text { (0 acres) }\end{array}$ & $\begin{array}{c}7 \\
(17.50)\end{array}$ & $\begin{array}{c}5 \\
(12.50)\end{array}$ & $\begin{array}{c}13 \\
(32.50)\end{array}$ & $\begin{array}{c}2 \\
(5.00)\end{array}$ & $\begin{array}{c}2 \\
(5.00)\end{array}$ & $\begin{array}{c}2 \\
(5.00)\end{array}$ & $\begin{array}{c}2 \\
(5.00)\end{array}$ & $\begin{array}{c}1 \\
(2.50)\end{array}$ & $\begin{array}{c}34 \\
(10.63)\end{array}$ \\
\hline $\begin{array}{c}\text { Marginal } \\
(0.1-2.5 \text { acres })\end{array}$ & $\begin{array}{c}16 \\
(40.00)\end{array}$ & $\begin{array}{c}23 \\
(57.50)\end{array}$ & $\begin{array}{c}18 \\
(45.00)\end{array}$ & $\begin{array}{c}9 \\
(22.50)\end{array}$ & $\begin{array}{c}15 \\
(37.50)\end{array}$ & $\begin{array}{c}16 \\
(40.00)\end{array}$ & $\begin{array}{c}10 \\
(25.00)\end{array}$ & $\begin{array}{c}9 \\
(22.50)\end{array}$ & $\begin{array}{c}116 \\
(36.25)\end{array}$ \\
\hline $\begin{array}{c}\text { Small } \\
(2.5-5.0 \text { acres })\end{array}$ & $\begin{array}{c}10 \\
(25.00)\end{array}$ & $\begin{array}{c}8 \\
(20.00)\end{array}$ & $\begin{array}{c}9 \\
(22.50)\end{array}$ & $\begin{array}{c}19 \\
(47.50)\end{array}$ & $\begin{array}{c}15 \\
(37.50)\end{array}$ & $\begin{array}{c}17 \\
(42.50)\end{array}$ & $\begin{array}{c}16 \\
(40.00)\end{array}$ & $\begin{array}{c}18 \\
(45.00)\end{array}$ & $\begin{array}{c}112 \\
(35.00)\end{array}$ \\
\hline $\begin{array}{c}\text { Medium } \\
(5.1-10.0 \text { acres })\end{array}$ & $\begin{array}{c}7 \\
(17.50)\end{array}$ & $\begin{array}{c}4 \\
(10.00)\end{array}$ & $\begin{array}{c}0 \\
(0.00)\end{array}$ & $\begin{array}{c}10 \\
(25.00)\end{array}$ & $\begin{array}{c}8 \\
(20.00)\end{array}$ & $\begin{array}{c}5 \\
(12.50)\end{array}$ & $\begin{array}{c}12 \\
(30.00)\end{array}$ & $\begin{array}{c}12 \\
(30.00)\end{array}$ & $\begin{array}{c}58 \\
(18.13)\end{array}$ \\
\hline
\end{tabular}

(Figures in parenthesis indicate percentage, $\mathrm{n}$ indicate sample size $)(\mathrm{P}$ value $=0.001)$ 
Table.5 Distribution of respondents according to their Livestock possession

\begin{tabular}{|c|c|c|c|c|c|c|c|c|c|}
\hline \multirow[t]{2}{*}{ Livestock Possesion } & \multicolumn{8}{|c|}{ Blocks } & \multirow[t]{2}{*}{ Pooled } \\
\hline & Budgam & Beerwah & Chadoora & Khansahib & Khag & B K Pora & Narbal & Nagam & \\
\hline $\mathbf{N}$ & 40 & 40 & 40 & 40 & 40 & 40 & 40 & 40 & 320 \\
\hline $\begin{array}{l}\text { Low livestock } \\
\text { possession(1-4 } \\
\text { livestock) }\end{array}$ & $\begin{array}{c}37 \\
(92.50)\end{array}$ & $\begin{array}{c}34 \\
(85.00)\end{array}$ & $\begin{array}{c}39 \\
(97.50)\end{array}$ & $\begin{array}{c}40 \\
(100.00)\end{array}$ & $\begin{array}{c}40 \\
(100.00)\end{array}$ & $\begin{array}{c}40 \\
(100.00)\end{array}$ & $\begin{array}{c}40 \\
(100.00)\end{array}$ & $\begin{array}{c}39 \\
(97.50)\end{array}$ & $\begin{array}{c}309 \\
(96.56)\end{array}$ \\
\hline $\begin{array}{l}\text { Medium livestock } \\
\text { possession(5-8 } \\
\text { livestock) }\end{array}$ & $\begin{array}{c}3 \\
(7.50)\end{array}$ & $\begin{array}{c}6 \\
(15.00)\end{array}$ & $\begin{array}{c}1 \\
(2.50)\end{array}$ & $\begin{array}{c}0 \\
(0.00)\end{array}$ & $\begin{array}{c}0 \\
(0.00)\end{array}$ & $\begin{array}{c}0 \\
(0.00)\end{array}$ & $\begin{array}{c}0 \\
(0.00)\end{array}$ & $\begin{array}{c}1 \\
(2.50)\end{array}$ & $\begin{array}{c}11 \\
(3.44)\end{array}$ \\
\hline
\end{tabular}

(Figures in parenthesis indicate percentage, $\mathrm{n}$ indicate sample size $)(\mathrm{P}$ value $=0.001)$

Communication patterns regarding zoonotic diseases among livestock farmers

Table.6 Contact with inter personal communication sources

\begin{tabular}{|c|c|c|c|c|c|c|c|c|c|}
\hline Communication Pattern & \multicolumn{7}{|c|}{ Blocks } \\
\hline & Budgam & Beerwah & Chadoora & Khansahib & Khag & B K Pora & Narbal & Nagam \\
\hline n & $\mathbf{4 0}$ & $\mathbf{4 0}$ & $\mathbf{4 0}$ & $\mathbf{4 0}$ & $\mathbf{4 0}$ & $\mathbf{4 0}$ & $\mathbf{4 0}$ & $\mathbf{4 0}$ & $\mathbf{3 2 0}$ \\
\hline Low (rare contact) & 1 & 1 & 1 & 1 & 1 & 0 & 1 & 2 & 8 \\
& $(0.79)$ & $(1.09)$ & $(1.10)$ & $(1.09)$ & $(1.17)$ & $(0.00)$ & $(0.40)$ & $(1.50)$ & $(0.89)$ \\
\hline Medium (occasionally contact) & 33 & 24 & 24 & 23 & 31 & 24 & 29 & 24 & 212 \\
& $(78.97)$ & $(51.64)$ & $(51.47)$ & $(51.09)$ & $(72.76)$ & $(50.91)$ & $(65.61)$ & $(53.93)$ & $(59.19)$ \\
\hline High(frequently contact) & 6 & 15 & 15 & 16 & 8 & 16 & 10 & 14 & 100 \\
& $(20.24)$ & $(47.27)$ & $(47.43)$ & $(47.83)$ & $(26.07)$ & $(49.09)$ & $(33.99)$ & $(44.57)$ & $(39.92)$ \\
\hline
\end{tabular}

(Figures in parenthesis indicate percentage, $\mathrm{n}$ indicate sample size) $(\mathrm{P}$ value $=0.403)$ 
Table .7 Mass media support with regard to zoonotic diseases

\begin{tabular}{|c|c|c|c|c|c|c|c|c|c|}
\hline \multirow[t]{2}{*}{ Mass media support } & \multicolumn{8}{|c|}{ Blocks } & \multirow[t]{2}{*}{ Pooled } \\
\hline & Budgam & Beerwah & Chadoora & Khansahib & Khag & B K Pora & Narbal & Nagam & \\
\hline $\mathbf{n}$ & 40 & 40 & 40 & 40 & 40 & 40 & 40 & 40 & 320 \\
\hline Low (rare) & $\begin{array}{c}38 \\
(95.00)\end{array}$ & $\begin{array}{c}39 \\
(97.50)\end{array}$ & $\begin{array}{c}35 \\
(87.50)\end{array}$ & $\begin{array}{c}38 \\
(95.00)\end{array}$ & $\begin{array}{c}36 \\
(90.00)\end{array}$ & $\begin{array}{c}37 \\
(92.50)\end{array}$ & $\begin{array}{c}35 \\
(87.50)\end{array}$ & $\begin{array}{c}37 \\
(92.50)\end{array}$ & $\begin{array}{c}295 \\
(92.19)\end{array}$ \\
\hline Medium (occasionally) & $\begin{array}{c}2 \\
(5.00)\end{array}$ & $\begin{array}{c}1 \\
(2.50)\end{array}$ & $\begin{array}{c}5 \\
(12.50)\end{array}$ & $\begin{array}{c}2 \\
(5.00)\end{array}$ & $\begin{array}{c}4 \\
(10.00)\end{array}$ & $\begin{array}{c}3 \\
(7.50)\end{array}$ & $\begin{array}{c}5 \\
(12.50)\end{array}$ & $\begin{array}{c}3 \\
(7.50)\end{array}$ & $\begin{array}{c}25 \\
(7.81)\end{array}$ \\
\hline
\end{tabular}

(Figures in parenthesis indicate percentage, $\mathrm{n}$ indicate sample size $)(\mathrm{P}$ value $=0.640)$

Knowledge level of livestock farmers regarding awareness of zoonotic diseases

Table. 8 Transmission of diseases from animals to human beings \& vice versa

\begin{tabular}{|c|c|c|c|c|c|c|c|c|c|}
\hline \multirow{2}{*}{ Transmission knowledge } & \multicolumn{8}{|c|}{ Blocks } & \multirow{2}{*}{ Pooled } \\
\hline & Budgam & Beerwah & Chadoora & Khansahib & Khag & B K Pora & Narbal & Nagam & \\
\hline $\mathbf{N}$ & 40 & 40 & 40 & 40 & 40 & 40 & 40 & 40 & 320 \\
\hline Aware & $\begin{array}{c}6 \\
(15.00)\end{array}$ & $\begin{array}{c}24 \\
(60.00)\end{array}$ & $\begin{array}{c}20 \\
(50.00)\end{array}$ & $\begin{array}{c}22 \\
(55.00)\end{array}$ & $\begin{array}{c}22 \\
(55.00)\end{array}$ & $\begin{array}{c}20 \\
(50.00)\end{array}$ & $\begin{array}{c}19 \\
(47.50)\end{array}$ & $\begin{array}{c}21 \\
(52.50)\end{array}$ & $\begin{array}{c}154 \\
(48.13)\end{array}$ \\
\hline Unaware & $\begin{array}{c}34 \\
(85.00)\end{array}$ & $\begin{array}{c}16 \\
(40.00)\end{array}$ & $\begin{array}{c}20 \\
(50.00)\end{array}$ & $\begin{array}{c}18 \\
(45.00)\end{array}$ & $\begin{array}{c}18 \\
(45.00)\end{array}$ & $\begin{array}{c}20 \\
(50.00)\end{array}$ & $\begin{array}{c}21 \\
(52.50)\end{array}$ & $\begin{array}{c}19 \\
(47.50)\end{array}$ & $\begin{array}{c}166 \\
(51.88)\end{array}$ \\
\hline
\end{tabular}

(Figures in parenthesis indicate percentage, $\mathrm{n}$ indicate sample size) $(\mathrm{P}$ value $=0.003)$

Table.9 Knowledge regarding transmission of various zoonotic diseases

\begin{tabular}{|c|c|c|c|c|c|c|c|c|c|}
\hline \multirow{2}{*}{ Disease Transmission } & \multicolumn{8}{|c|}{ Blocks } & \multirow{2}{*}{ Pooled } \\
\hline & Budgam & Beerwah & Chadoora & Khansahib & Khag & B K Pora & Narbal & Nagam & \\
\hline $\mathbf{n}$ & 40 & 40 & 40 & 40 & 40 & 40 & 40 & 40 & 320 \\
\hline Agree & $\begin{array}{c}12 \\
(30.00)\end{array}$ & $\begin{array}{c}5 \\
(12.50)\end{array}$ & $\begin{array}{c}5 \\
(12.50)\end{array}$ & $\begin{array}{c}5 \\
(12.50)\end{array}$ & $\begin{array}{c}6 \\
(15.00)\end{array}$ & $\begin{array}{c}6 \\
(15.00)\end{array}$ & $\begin{array}{c}8 \\
(20.00)\end{array}$ & $\begin{array}{c}4 \\
(10.00)\end{array}$ & $\begin{array}{c}51 \\
(15.94)\end{array}$ \\
\hline Disagree & $\begin{array}{c}25 \\
(62.50)\end{array}$ & $\begin{array}{c}28 \\
(70.00)\end{array}$ & $\begin{array}{c}26 \\
(65.00)\end{array}$ & $\begin{array}{c}30 \\
(75.00)\end{array}$ & $\begin{array}{c}31 \\
(77.50)\end{array}$ & $\begin{array}{c}24 \\
(60.00)\end{array}$ & $\begin{array}{c}26 \\
(65.00)\end{array}$ & $\begin{array}{c}30 \\
(75.00)\end{array}$ & $\begin{array}{c}220 \\
(68.75)\end{array}$ \\
\hline Don't know & $3(7.50)$ & $7(17.50)$ & $9(22.50)$ & $5(12.50)$ & $3(7.50)$ & $10(25.00)$ & $6(15.00)$ & $6(15.00)$ & $49(15.31)$ \\
\hline
\end{tabular}

(Figures in parenthesis indicate percentage, $\mathrm{n}$ indicate sample size) $(\mathrm{P}$ value $=0.297)$ 
Table.10 Knowledge regarding possible means of transmission of zoonotic diseases

\begin{tabular}{|c|c|c|c|c|c|c|c|c|c|}
\hline \multirow[t]{2}{*}{ Means of transmission } & \multicolumn{8}{|c|}{ Blocks } & \multirow[t]{2}{*}{ Pooled } \\
\hline & Budgam & Beerwah & Chadoora & Khansahib & Khag & B K Pora & Narbal & Nagam & \\
\hline $\mathbf{N}$ & 40 & 40 & 40 & 40 & 40 & 40 & 40 & 40 & 320 \\
\hline Agree & $\begin{array}{c}1 \\
(2.50)\end{array}$ & $\begin{array}{c}8 \\
(20.00)\end{array}$ & $\begin{array}{c}8 \\
(20.00)\end{array}$ & $\begin{array}{c}8 \\
(20.00)\end{array}$ & $\begin{array}{c}10 \\
(25.00)\end{array}$ & $\begin{array}{c}7 \\
(17.50)\end{array}$ & $\begin{array}{c}3 \\
(7.50)\end{array}$ & $\begin{array}{c}4 \\
(10.00)\end{array}$ & $\begin{array}{c}49 \\
(15.31)\end{array}$ \\
\hline Disagree & $\begin{array}{c}18 \\
(45.00)\end{array}$ & $\begin{array}{c}23 \\
(57.50)\end{array}$ & $\begin{array}{c}18 \\
(45.00)\end{array}$ & $\begin{array}{c}20 \\
(50.00)\end{array}$ & $\begin{array}{c}25 \\
(62.50)\end{array}$ & $\begin{array}{c}27 \\
(67.50)\end{array}$ & $\begin{array}{c}30 \\
(75.00)\end{array}$ & $\begin{array}{c}28 \\
(70.00)\end{array}$ & $\begin{array}{c}189 \\
(59.06)\end{array}$ \\
\hline Don't know & $\begin{array}{c}21 \\
(52.50)\end{array}$ & $\begin{array}{c}9 \\
(22.50)\end{array}$ & $\begin{array}{c}14 \\
(35.00)\end{array}$ & $\begin{array}{c}12 \\
(30.00)\end{array}$ & $\begin{array}{c}5 \\
(12.50)\end{array}$ & $\begin{array}{c}6 \\
(15.00)\end{array}$ & $\begin{array}{c}7 \\
(17.50)\end{array}$ & $\begin{array}{c}8 \\
(20.00)\end{array}$ & $\begin{array}{c}82 \\
(25.63)\end{array}$ \\
\hline
\end{tabular}

(Figures in parenthesis indicate percentage, $\mathrm{n}$ indicate sample size) $(\mathrm{P}$ value $=0.001)$

Table.11 Knowledge regarding risks of zoonoses from various actions/ activities related with livestock rearing

\begin{tabular}{|c|c|c|c|c|c|c|c|c|c|}
\hline \multirow{2}{*}{$\begin{array}{l}\text { Actions/ } \\
\text { Activities }\end{array}$} & \multicolumn{8}{|c|}{ Blocks } & \multirow[t]{2}{*}{ Pooled } \\
\hline & Budgam & Beerwah & Chadoora & Khansahib & Khag & B K Pora & Narbal & Nagam & \\
\hline $\mathbf{n}$ & 40 & 40 & 40 & 40 & 40 & 40 & 40 & 40 & 320 \\
\hline Agree & $\begin{array}{c}18 \\
(45.00)\end{array}$ & $\begin{array}{c}12 \\
(30.00)\end{array}$ & $\begin{array}{c}15 \\
(37.50)\end{array}$ & $\begin{array}{c}22 \\
(55.00)\end{array}$ & $\begin{array}{c}15 \\
(37.50)\end{array}$ & $\begin{array}{c}8 \\
(20.00)\end{array}$ & $\begin{array}{c}18 \\
(45.00)\end{array}$ & $\begin{array}{c}12 \\
(30.00)\end{array}$ & $\begin{array}{c}120 \\
(37.50)\end{array}$ \\
\hline Disagree & $\begin{array}{c}22 \\
(55.00)\end{array}$ & $\begin{array}{c}28 \\
(70.00)\end{array}$ & $\begin{array}{c}25 \\
(62.50)\end{array}$ & $\begin{array}{c}18 \\
(45.00)\end{array}$ & $\begin{array}{c}25 \\
(62.50)\end{array}$ & $\begin{array}{c}32 \\
(80.00)\end{array}$ & $\begin{array}{c}22 \\
(55.00)\end{array}$ & $\begin{array}{c}28 \\
(70.00)\end{array}$ & $\begin{array}{c}200 \\
(62.50)\end{array}$ \\
\hline
\end{tabular}

(Figures in parenthesis indicate percentage, $\mathrm{n}$ indicate sample size $)(\mathrm{P}$ value $=0.046)$

Table.12 Knowledge regarding zoonotic diseases via sale of diseased animals

\begin{tabular}{|c|c|c|c|c|c|c|c|c|c|}
\hline \multirow{2}{*}{$\begin{array}{c}\text { Transmission via sale of } \\
\text { diseased animals }\end{array}$} & \multicolumn{8}{|c|}{ Blocks } & \multirow[t]{2}{*}{ Pooled } \\
\hline & Budgam & Beerwah & Chadoora & Khansahib & Khag & B K Pora & Narbal & Nagam & \\
\hline $\mathbf{N}$ & 40 & 40 & 40 & 40 & 40 & 40 & 40 & 40 & 320 \\
\hline Unaware & $\begin{array}{c}25 \\
(62.50)\end{array}$ & $\begin{array}{c}19 \\
(47.50)\end{array}$ & $\begin{array}{c}18 \\
(45.00)\end{array}$ & $\begin{array}{c}17 \\
(42.50)\end{array}$ & $\begin{array}{c}22 \\
(55.00)\end{array}$ & $\begin{array}{c}21 \\
(52.50)\end{array}$ & $\begin{array}{c}17 \\
(42.50)\end{array}$ & $\begin{array}{c}20 \\
(50.00)\end{array}$ & $\begin{array}{c}159 \\
(49.69)\end{array}$ \\
\hline
\end{tabular}

(Figures in parenthesis indicate percentage, $\mathrm{n}$ indicate sample size) $(\mathrm{P}$ value $=0.625)$ 
Extent of knowledge among livestock farmers with regard to risks of zoonotic diseases scenario

Regarding importance of zoonotic hazards, respondents in this study revealed that $(48.13 \%)$ and found that there is a significant variation with regard to transmission of diseases that can occur between vertebrate animals and human beings. When compared with the study of Hundal et al., (2016) which he and his co-workers found that most of the zoonotic diseases are transmitted from animals. From the present study it clearly indicates a relatively lower level of awareness among the respondents. This low level of awareness is likely to expose them to an increased risk of contracting zoonotic diseases.

Knowledge regarding various zoonotic diseases was taken into consideration and found that $(15.94 \%)$ agree that diseases can be transmitted from animals to human beings and there is non-significant difference in different blocks. Also knowledge level of livestock farmers was checked with regard to zoonotic diseases that may be transmitted by consumption of contaminated milk, meat and eggs, through aerosol, through contaminated water or feed and also with contact with infected animals and from the study it was revealed that only $(15.31 \%)$ agree that above stated modes were the means of transmission of zoonotic diseases.

Similar levels of knowledge were also reported by Tebug et.al (2014) regarding transmission of zoonotic diseases and also compared with the study of Chengula et al., (2013) found that eating of dead and improperly cooked meat together with unboiled milk, rotten eggs were the main modes of transmission of zoonotic diseases. The knowledge regarding risks of zoo noses from various actions/ activities related with livestock rearing viz. walking bare feet in the animal shed, slaughtering animals, skin-skin contact with animals, preparing skin, disposing off manure and cutting off road side pasture and it was found that only (37.50\%) of the respondents agree and showed a significant difference in different blocks that from above activities, there were increased chances of zoonotic diseases.

Revealed by the Lean et al., (2014) that the highest risk of the zoonotic diseases was the milking shed, exposure of urine splashes, disposing off manure were the main sources of zoonotic diseases. Also (50.31\%) agree that zoonotic diseases transmission occurs via. Selling/ slaughtering of diseased animals. When compared with study of Pal et al., (2013) assessed that slaughtering or processing of skin can lead to transmission of zoonotic diseases. From overall study it was revealed that knowledge and awareness regarding transmission, modes of transmission and risks related to zoonotic diseases was found limited at some levels. This stressed on the need for providing better knowledge for effective control of zoonosis.

\section{References}

Ahn B Y, Kim J and Lee Y. 1981. Survey on consumers attitude and consumption pattern of fresh and processed meat. Korean Journal of Animal Sciences, 23(1): 62-67.

Asbjer E. 2009. Dog population management in Malawi and Peri. Project report, Department of Biomedical Sciences and Veterinary Public Health. Swedish University of Agricultural Sciences, International Journal of Recent Scientific Research, 6: 5131-5138.

Chengula A, Mdegela R H and Kasanga C. J. 2013. Attitude, knowledge and practices of pastoral list and agro pastoralists towards livestock diseases affecting 
domestic animals in Arusha, Manyara and Morogoro regions, Tanzania. Journal of Health, Medicine and Nursing, 1: 13-19.

Folayan J A. 2013. Socio- economic analysis of fadama farmers in Akure South local government area of Ondo State, Nigeria. American Journal of Humanities and Social Sciences, 1(1): 10-17.

Gamit M P, Rani V D, Bhabho I N, Tyagi K $\mathrm{K}$ and Rathod A D. 2015. Entrepreneurial behaviour of dairy farmers in Surat district of South Gujarat. International Journal of Advanced Multidisciplinary Research, 2(8): 50-56.

Gummow B. 2003.A survey of zoonotic diseases contracted by South African veterinarians. Journal of the South African Veterinary Association, 74(3): 72-76.

Huang L C, Goh, Y N and Mohaidin Z. 2014. Factors influencing consumer intentions to avoid broiler chicken meat and products in Malaysia. International Food Research Journal, 21(1): 181-188.

Hundal J S, Sodhi S S, Gupta A, Singh J and Chahal U S. 2016. Awareness, knowledge, and risks of zoonotic diseases among livestock farmers in Punjab, Veterinary World, 9(2): 186191.

Lean M M, Ruscoe Q, Kline T, King C and Nesdale, A. 2014. A cluster of three cases of leptospirosis in dairy farm workers in New Zealand. The New Zealand Medical Journal, 127(1388): 13-20.

Mahdi N $\mathrm{K}$ and Ali N $\mathrm{H}$. 2002.Crytosporiodiosis among animal handlers and their livestock in Basrah, Iraq. East African Medical Journal, 79(10): 550-553.

Niyaki S A N and Allahyari M S. 2010. Socio-personal characteristics on the adoption of rice fish culture system in North of Iran. African Journal of Agricultural Research, 5(24): 34703476.

Nkuchia, M M, Ruth L, Chris A B and Henriette, V. 2007. Infectious diseases surveillance. Blackwell Publishing, 2: 246-248.

Oluwafemi, R A. 2015. Socio-economic characteristics of Village poultry farmers in Nigeria and their Production constraints. International Journal of Life Science and Engineering, 1(4): 132139.

Pal M, Tesfaye S and Dave P. 2013. Zoonoses occupationally acquired by abattoir workers. Journal of Environment and occupational science, 2(3): 155-162.

Rahman S and Gupta J. 2014. Knowledge and adoption level of improved dairy farming practices of SHG members and non-members in Kamrup district of Assam. Indian Journal of Animal Research, 49 (2): 234-240.

Rathod P K, Landge S, Nikam T R and Vajreshwari S. 2011. Socio-personal profile and constraints of dairy farmers. Karnataka Journal of Agricultural. Sciences, 24(4): 619-621.

Sathyanarayan K, Jagadeeswary V, Murthy V C, Ruban S W and Sudha G. 2010. Socio-economic status of livestock farmers of Narasapura Village. Veterinary World, 3(5): 215-218.

Smiths H L and Cutler S J. 2004. Contributions of Biotechnology to the control and prevention of brucellosis in Africa . African Journal of Biotechnology, 3: 631-639.

Tebug S F, Njunga G R, Chagunda, M G G, Mapemba J P, Awah-Ndukum J and Wiedemann S. 2014. Risk, knowledge and preventive measures of smallholder dairy farmers in northern Malawi with regard to zoonotic brucellosis and bovine tuberculosis. Onderstepoort 
Journal of Veterinary Research.,81: 16.

Ward R. and Moon, W. 1996. Changing attitudes about fat and cholesterol: Do they impact meat consumption choices. Magazine Food Farm Resources Issues, 3: 40-42.

Woolhouse M E J and Gowtage - Sequeria S. 2005. Host range and emerging and re- emerging pathogens. Emerging Infectious Diseases, 11: 1842-1847. Yadav S P, Mathur M L and Dixit A K. 2006. Knowledge and attitude towards tuberculosis among sandstone quarry workers in desert parts of Rajasthan. Indian Journal of Tuberculosis, 53:187195.

\section{How to cite this article:}

Insha Mir and Abdul Hai Bhat. 2020. Knowledge and Risks of Zoonotic Diseases among Livestock Farmers in Budgam District of Kashmir Valley, India. Int.J.Curr.Microbiol.App.Sci. 9(04): 2924-2934. doi: https://doi.org/10.20546/ijcmas.2020.904.342 